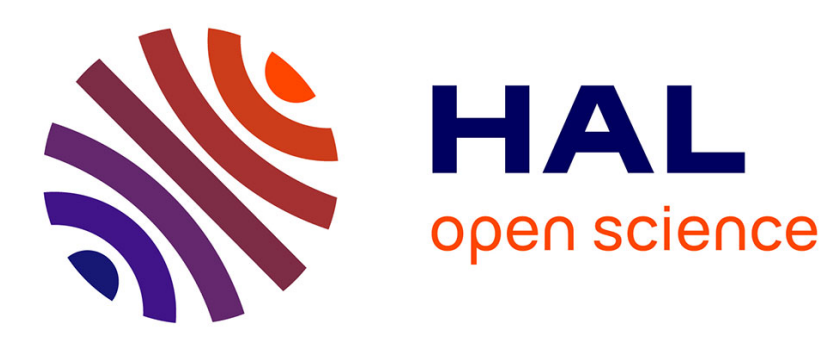

\title{
An EWMA signed ranks control chart with reliable run length performances
}

Theodoros Perdikis, Stelios Psarakis, Philippe Castagliola, Petros Maravelakis

\section{To cite this version:}

Theodoros Perdikis, Stelios Psarakis, Philippe Castagliola, Petros Maravelakis. An EWMA signed ranks control chart with reliable run length performances. Quality and Reliability Engineering International, 2021, 37 (3), pp.1266-1284. 10.1002/qre.2795 . hal-03163631

\section{HAL Id: hal-03163631 \\ https://hal.science/hal-03163631}

Submitted on 20 Aug 2021

HAL is a multi-disciplinary open access archive for the deposit and dissemination of scientific research documents, whether they are published or not. The documents may come from teaching and research institutions in France or abroad, or from public or private research centers.
L'archive ouverte pluridisciplinaire HAL, est destinée au dépôt et à la diffusion de documents scientifiques de niveau recherche, publiés ou non, émanant des établissements d'enseignement et de recherche français ou étrangers, des laboratoires publics ou privés. 


\title{
An EWMA Signed Ranks Control Chart with Reliable Run Length Performances
}

\author{
Theodoros Perdikis ${ }^{1}$, Stelios Psarakis*1, \\ Philippe Castagliola ${ }^{2}$, and Petros E. Maravelakis ${ }^{3}$ \\ ${ }^{1}$ Department of Statistics \& Laboratory of Statistical Methodology, \\ Athens University of Economics and Business Athens, Greece \\ ${ }^{2}$ Université de Nantes \& LS2N UMR CNRS 6004, Nantes, France \\ ${ }^{3}$ Department of Business Administration, University of Piraeus, \\ Piraeus, Greece
}

August 20, 2021

\begin{abstract}
During the design phase of a control chart, the determination of its exact Run Length properties plays a vital role for its optimal operation. Markov chain or integral equation methods have been extensively applied in the design phase of conventional control charts. However, for distribution-free schemes, due to the discrete nature of the statistics being used (such as the Sign or the Wilcoxon Signed Rank statistics, for instance), it is impossible to accurately compute their Run Length properties. In this work, a modified distribution-free Phase II EWMA-type chart based on the Wilcoxon Signed Rank statistic is considered and its exact Run Length properties are discussed. A continuous transformation of the Wilcoxon Signed Rank statistic, combined with the classical Markov Chain method, is used for the determination of the Average Run Length in the in- and out-of control cases. Moreover, its exact performance is derived without any knowledge of the distribution of sample observations. Finally, an illustrative example is provided showing the practical implementation of our proposed chart.
\end{abstract}

Keywords:Nonparametric control chart; Wilcoxon signed rank statistic;Markov chain; EWMA control chart.

\section{Introduction}

SPM (Statistical Process Monitoring) techniques are of high importance for manufacturing and non-manufacturing applications as they aim to identify the presence of possible assignable causes in processes. Control charts are one of the major tools of SPM. They are on-line process monitoring techniques whose

*psarakis@aueb.gr(corresponding author) 
purpose is to detect shifts in processes as fast as possible. One of the most commonly used type of control chart is the Shewhart-type control chart (see Shewhart $^{1}$ ). A significant improvement in charting techniques was the introduction of memory-type methods like the EWMA (Exponentially Weighted Moving Average) by Roberts ${ }^{2}$ and the CUSUM (Cumulative Sum) by Page ${ }^{3}$. It is well known that Shewhart-type control charts are preferable in cases where large mean shifts need to be detected. On the other hand, EWMA and CUSUM schemes are able to detect small to moderate shifts in processes, faster than conventional standard control charts, due to the fact that, at each sampling point, they take into account previous measurements.

In general, during the design phase of a measurement control chart, its design parameters are obtained based on the assumption that the observations, collected over time, are normally distributed random variables or, at least, they follow some known distribution. However, in practice, this assumption is commonly violated, since the underlying distribution of the characteristic to be monitored is generally unknown. As a result, a new class of monitoring schemes called distribution-free (or nonparametric) control schemes has been introduced into the literature. For nonparametric control charts, the chart's design parameters as well as the Run Length properties are derived based on the distribution of the corresponding nonparametretric statistics which are used (such us the Sign or the Wilcoxon Signed Rank statistics) with only a minimal knowledge of the form of the underlying distribution for the characteristic to be monitored. For a comprehensive overview of the literature regarding the design and operations of several univariate and multivariate nonparametric control charts the reader is advised to refer to Chakraborti et al. ${ }^{4}$, Qiu ${ }^{5}$, Chakraborti and Graham ${ }^{6}$. Concerning distribution-free EWMA control charts, two of the most commonly used nonparametric statistics that have been used and presented into the literature are the Sign statistic (Amin and Searcy ${ }^{7}$, Graham et al. ${ }^{8}$, Yang et al. ${ }^{9}$, Aslam et al. $\left.{ }^{10}, \mathrm{Riaz}^{11}, \mathrm{Lu}^{12}, \mathrm{Haq}^{13}\right)$ and the Wilcoxon Signed Rank or Rank-Sum statistics (Li et al. ${ }^{14}$, Graham et al. ${ }^{15}$, Chakraborty et al. ${ }^{16}$, Abid et al. $\left.{ }^{17}\right)$. Additionally, recent developments of distribution- free EWMA-type schemes can be found at Raza et al. ${ }^{18}$, Alevizakos et al. ${ }^{19}$, Mabude et al. ${ }^{20}$.

The use of reliable metrics that can efficiently measure the performance of a control scheme plays a vital role in its design phase. One of the most widely used technique is to examine the performance of a control chart by evaluating its in- and out-of- control RL (Run Length) properties such as the ARL (Average Run Length), SDRL (Standard Deviation Run Length) or quantile-type metrics like the MRL (Median Run Length) or the $\mathrm{RL}_{0.95}$ (0.95-quantile of the $\mathrm{RL}$ ). For EWMA and CUSUM-type schemes their RL properties are often obtained by using the Markov Chain approach of Brook and Evans ${ }^{21}$ which is based on the discretization of the control limits interval into sub-intervals. It should be noted that, regarding the design phase of an EWMA-type control chart a proper computation of its RL properties is essential. Specifically, for the determination of the design parameters $\lambda$ (smoothing parameter) and $K$ (control limit parameter) of an EWMA-type chart, a searching algorithm needs to be conducted, for a particular shift in the process, in order to find the optimal pair $\left(\lambda^{*}, K^{*}\right)$ which minimizes the out-of-control ARL, under the constraint $\mathrm{ARL}=\mathrm{ARL}_{0}$ where $\mathrm{ARL}_{0}$ is some predefined value for the in-control ARL. In the case of measure- 
ment data (usually assuming normality) as the number of sub-intervals increases the method proposed by Brook and Evans ${ }^{21}$ tends to give reliable approximations of the chart's RL properties. However, in a nonparametric context, due to the discrete nature of the statistics that is being used (for instance, the Sign or Wilcoxon Signed Rank statistics), it is not feasible to exactly compute the in- $\left(\mathrm{ARL}_{0}\right)$ and out-of-control $\left(\mathrm{ARL}_{1}\right)$ ARL by using this approach (see Weiß ${ }^{22}$ for instance). As it will be shown in the rest of the paper, the corresponding ARL values are actually highly affected by the number of sub-intervals. As a consequence, robust methodologies are essential for the proper computation of its RL properties. Castagliola et al. ${ }^{23}$ based on the approach of Rakitzis et al. ${ }^{24}$ introduced a new class of distribution-free EWMA-type chart for count data (CEWMA SN chart) in which a proper discrete Markov-chain approach was used for the determination of the ARL for the in- and out-of control cases. Tang et al. ${ }^{25}$ using the same discrete Markov method extended the CEWMA SN chart by adding an adaptive feature in the smoothing parameter. Recently, Wu et al. ${ }^{26}$ proposed a distribution-free EWMA-TBEA (Time Between Events and Amplitude) control chart where they introduced a new approach called as the "continuousify" method in which the values of the initial discrete random variables are transformed into continuous ones based on weighted Gaussian Kernels. As a result, since the Markov chain of Brook and Evans ${ }^{21}$ performs well in the case of continuous random variables, $\mathrm{Wu}$ et al. ${ }^{26}$ showed that the above method yields robust results without the need of setting large values for the number sub-intervals used in the Markov chain. In this paper, based on the approach of $\mathrm{Wu}$ et al. ${ }^{26}$ we will introduce a distribution-free one-sided EWMA control chart based on the Wilcoxon Signed Rank statistic with robust ARL results regardless of the underlying distribution of the observations. It should be noted that, this work aims to provide a methodology which guarantees stable results in the chart's RL properties.

The paper is organised as follows: In Section 2, a modified version of the nonparametric EWMA chart based on the Wilcoxon Signed Rank statistic proposed by Graham et al. ${ }^{15}$ (denoted as the WSR EWMA chart) is proposed. Moreover, an extension of the former control chart (denoted as the C-WSR EWMA chart) is presented in which the Gaussian Kernel estimation approach of $\mathrm{Wu}$ et al. ${ }^{26}$ is used. In Section 3, a numerical analysis is conducted concerning the robustness of the "continuousify" method and optimal design parameters $\left(\lambda^{*}, K^{*}\right)$ are obtained under several shifts and sample sizes. Additionally, the efficiency of the "continuousify" method is examined for several Kernel density functions. In Section 4 an illustrative example is discussed to show the practical implementation of the operation of our proposed chart. Finally, in Section 5, some concluding remarks and suggestions for future works are discussed.

\section{The distribution free one-sided WSR EWMA chart}

Graham et al. ${ }^{15}$ introduced a new nonparametric two-sided EWMA chart based on the Wilcoxon Signed Rank statistic (NPEWMA-SR chart). Using the MarkovChain approach of Brook and Evans ${ }^{21}$ they obtained its optimal design param- 
eters and they examined its out-of-control performance under several symmetric distributions. In this current work, we present a modified version of the NPEWMA-SR chart, providing its Run Length properties for both in- and outof-control conditions regardless of the observations' underlying distribution. We will only focus on the design of an EWMA scheme capable of detecting increases in the product's characteristic. As mentioned above, this paper does not aim to investigate the performance of a new EWMA-type scheme based on Signed Ranks but to present an improved and efficient approach to compute its Run Length properties. In this Section, a brief review of the Wilcoxon Signed Rank statistic will be firstly presented and then our proposed modified version of the EWMA control chart based on signed ranks will be introduced.

\subsection{Theoretical background of the Wilcoxon Signed Rank statistic}

The Wilcoxon Signed Rank test statistic is one of the most commonly used nonparametric technique for testing hypotheses about a location parameter, $\theta_{0}$, of a symmetric continuous distribution. Suppose that at each sampling point $t=1,2, \ldots$ a subgroup $\left\{X_{t, 1}, X_{t, 2}, \ldots, X_{t, n}\right\}$ of size $n$ is collected following an unknown continuous symmetric distribution with c.d.f. (cumulative distribution function) $F_{X}(x \mid \theta)$ where $\theta$ is the location parameter to be monitored (assumed as known). With respect to the Phase II implementation of the control chart, when the process is in-control, assuming $\theta$ as the median of the process we have that $\mathrm{P}\left(X_{t, j}>\theta_{0} \mid \theta=\theta_{0}\right)=\mathrm{P}\left(X_{t, j}<\theta_{0} \mid \theta=\theta_{0}\right)=p_{0}=0.5$. On the other hand, let $p_{1}=\mathrm{P}\left(X_{t, j}>\theta_{0} \mid \theta=\theta_{1}\right)=1-F_{X}\left(\theta_{0} \mid \theta_{1}\right)$ be defined as the probability of having an observation larger than $\theta_{0}$ when the process runs out-of-control with median $\theta=\theta_{1}$. By definition, the Wilcoxon signed rank statistic $\mathrm{SR}_{t}$ is equal to:

$$
\mathrm{SR}_{t}=\sum_{j=1}^{n} \operatorname{sign}\left(X_{t, j}-\theta_{0}\right) L_{t, j},
$$

where $\operatorname{sign}(x)=-1,0$ or +1 if $x<0, x=0$ or $x>0$, respectively. Additionally, $L_{t, j} \in\{1,2, \ldots, n\}$ denotes the rank of the absolute value of the differences $\left|X_{t, j}-\theta_{0}\right|, j=1,2, \ldots, n$ for subgroup $t=1,2, \ldots$ Therefore, $\mathrm{SR}_{t}$ is the sum of the signed ranks defined on $\left\{-\frac{n(n+1)}{2},-\frac{n(n+1)}{2}+2, \ldots, \frac{n(n+1)}{2}-2, \frac{n(n+1)}{2}\right\}$. Moreover, the statistic $\mathrm{SR}_{t}$ can be alternatively expressed as:

$$
\mathrm{SR}_{t}=2 \mathrm{SR}_{t}^{+}-\frac{n(n+1)}{2},
$$

where $\mathrm{SR}_{t}^{+}$is the sum of the positive ranks. More details regarding the properties and alternative expressions of $\mathrm{SR}_{t}^{+}$can be found in Gibbons and Chakraborti ${ }^{27}$. Under the null hypothesis (i.e. for $p=p_{0}$ ), McCornack $^{28}$ provided a methodology in which the p.m.f. (probability mass function) $f_{\mathrm{SR}_{t}^{+}}(s \mid n)$ of $\mathrm{SR}_{t}^{+}$can be obtained exactly, without any approximation, through the evaluation of the number $N_{\mathrm{SR}_{t}^{+}}(s \mid n)$ of subsets of integers in $\{1, \ldots, n\}$ having a sum equal to $s \in\left\{0,1, \ldots, \frac{n(n+1)}{2}\right\}$, i.e.

$$
N_{\mathrm{SR}_{t}^{+}}(s \mid n)=N_{\mathrm{SR}_{t}^{+}}(s \mid n-1)+N_{\mathrm{SR}_{t}^{+}}(s-n \mid n-1),
$$


and by computing

$$
f_{\mathrm{SR}_{t}^{+}}(s \mid n)=\frac{N_{\mathrm{SR}_{t}^{+}}(s \mid n)}{2^{n}} .
$$

Concerning the computation of the p.m.f. of $\mathrm{SR}_{t}$ under the alternative hypothesis (i.e. $p \neq p_{0}$ ), the p.m.f. $f_{\mathrm{SR}_{t}^{+}}(s \mid n, p)$ of $\mathrm{SR}_{t}^{+}$can be obtained by evaluating firstly its p.g.f. (probability generating function) $G_{\mathrm{SR}_{t}^{+}}(\omega \mid n, p)$ (Bennett ${ }^{29}$ ):

$$
G_{\mathrm{SR}_{t}^{+}}(\omega \mid n, p)=\prod_{i=1}^{n}\left(p \omega^{i}+q\right)
$$

where $q=1-p$. More specifically, the p.m.f. $f_{\mathrm{SR}_{t}^{+}}(s \mid n, p)$ of $\mathrm{SR}_{t}^{+}$will be obtained by differentiating $G_{\mathrm{SR}_{t}^{+}}(\omega \mid n, p), s$ times, for $\omega=0$, using the formula

$$
f_{\mathrm{SR}_{t}^{+}}(s \mid n, p)=\left.\frac{1}{s !} G_{\mathrm{SR}_{t}^{+}}^{(s)}(\omega \mid n, p)\right|_{\omega=0},
$$

where $G_{\mathrm{SR}_{t}^{+}}^{(s)}(\omega \mid n, p)$ is the $s^{\text {th }}$ derivative of $G_{\mathrm{SR}_{t}^{+}}(\omega \mid n, p)$ and $G_{\mathrm{SR}_{t}^{+}}(\omega \mid n, p)$ is a polynomial of degree $\frac{n(n+1)}{2}$. As a result for $s \in\left\{0,1, \ldots, \frac{n(n+1)}{2}\right\}, G_{\mathrm{SR}_{t}^{+}}^{(s)}(\omega \mid n, p)$ is also a polynomial of degree $\frac{n(n+1)}{2}-s$ which can be expressed as:

$$
\frac{1}{s !} G_{\mathrm{SR}_{t}^{+}}^{(s)}(\omega \mid n, p)=\sum_{j=0}^{\frac{n(n+1)}{2}-s} c_{s, j} w^{j},
$$

where $c_{s, j}$ is the coefficient of degree $j$ corresponding to the polynomial $\frac{1}{s !} G_{\mathrm{SR}_{t}^{+}}^{(s)}(\omega \mid n, p)$. It should be noted that this method can also be applied for the null hypothesis. Moreover, as polynomials can be easily coded with real valued vectors, fast arithmetic operations (addition, multiplication and power) and derivation can be efficiently implemented with programming languages like Matlab or Python, thus providing a very fast computation of $f_{\mathrm{SR}_{t}^{+}}(s \mid n, p)$ for any value of $p$.

\subsection{The upper-sided WSR EWMA chart}

The upper-sided EWMA chart based on Signed Ranks (WSR EWMA chart) is defined by the following recursive formula:

$$
Z_{t}=\max \left(0, \lambda \mathrm{SR}_{t}+(1-\lambda) Z_{t-1}\right), Z_{0}=0,
$$

with a fixed asymptotic upper control limit defined as:

$$
\mathrm{UCL}=\mathrm{E}\left(\mathrm{SR}_{t}\right)+K \sqrt{\mathrm{V}\left(\mathrm{SR}_{t}\right)} \times \sqrt{\frac{\lambda}{2-\lambda}} .
$$

Using the relationship between $\mathrm{SR}_{t}$ and $\mathrm{SR}_{t}^{+}$, presented in (1), the in-control expected value and variance of $\mathrm{SR}_{t}$ are equal to:

$$
\begin{aligned}
\mathrm{E}\left(\mathrm{SR}_{t}\right) & =\frac{n(n+1)\left(2 p_{0}-1\right)}{2}, \\
\mathrm{~V}\left(\mathrm{SR}_{t}\right) & =\frac{2 n(n+1)(2 n+1) p_{0}\left(1-p_{0}\right)}{3} .
\end{aligned}
$$


where $p_{0}$ is the in control value. If we assume that $\theta$ is the median (i.e. $p_{0}=0.5$ ) we simply have $\mathrm{E}\left(\mathrm{SR}_{t}\right)=0$ and $\mathrm{V}\left(\mathrm{SR}_{t}^{+}\right)=\frac{n(n+1)(2 n+1)}{6}$. It should be noted that, the upper-sided WSR EWMA chart, besides monitoring the median, can also be used for monitoring any percentile defined on $p_{0} \in(0,1)$.

In order to obtain the zero-state ARL and SDRL of the WSR EWMA control chart, the efficiency of the standard approach proposed by Brook and Evans ${ }^{21}$ will be tested, which assumes that the operation of this control chart can be well represented by a discrete-time Markov chain with $m+2$ states. Specifically, the transition probability matrix $\mathbf{P}$ will be defined as:

$$
\mathbf{P}=\left(\begin{array}{cc}
\mathbf{Q} & \mathbf{r} \\
\mathbf{0}^{\boldsymbol{\top}} & 1
\end{array}\right)=\left(\begin{array}{cccccc}
Q_{0,0} & Q_{0,1} & \ldots & Q_{0, m-1} & Q_{0, m} & r_{0} \\
Q_{1,0} & Q_{1,1} & \ldots & Q_{1, m-1} & Q_{1, m} & r_{1} \\
\vdots & \vdots & \ddots & \vdots & \vdots & \vdots \\
Q_{m, 0} & Q_{m, 1} & \ldots & Q_{m, m-1} & Q_{m, m} & r_{m} \\
0 & 0 & \ldots & 0 & 0 & 1
\end{array}\right)
$$

where $\mathbf{Q}$ is the $(m+1, m+1)$ matrix of transient probabilities, $\mathbf{0}^{\mathbf{\top}}=(0,0, \ldots, 0)$ and $\mathbf{r}=\mathbf{1}-\mathbf{Q} \mathbf{1}$. In addition, the transient probabilities, $Q_{k, i}$ will be computed as:

- if $i=0$,

$$
Q_{k, 0}=F_{\mathrm{SR}_{t}}\left(-\frac{(1-\lambda) H_{k}}{\lambda} \mid n, p_{1}\right)
$$

- if $i=1,2, \ldots, m$,

$$
Q_{k, i}=F_{\mathrm{SR}_{t}}\left(\frac{H_{i}+\Delta-(1-\lambda) H_{k}}{\lambda} \mid n, p_{1}\right)-F_{\mathrm{SR}_{t}}\left(\frac{H_{i}-\Delta-(1-\lambda) H_{k}}{\lambda} \mid n, p_{1}\right) .
$$

where $F_{\mathrm{SR}_{t}}\left(x \mid n, p_{1}\right)=\sum_{s=0}^{x} f_{\mathrm{SR}_{t}}\left(s \mid n, p_{1}\right)$ and $f_{\mathrm{SR}_{t}}\left(x \mid n, p_{1}\right)$ are the c.d.f. and p.m.f. of $\mathrm{SR}_{t}$ which both depend on the sample size $n$ and shift $p_{1}$. Let $\mathbf{q}=\left(q_{0}, q_{1}, \ldots, q_{m}\right)^{\top}$ be the $(m+1,1)$ vector of initial probabilities associated with the $m+1$ transient states. In our case, we assume $\mathbf{q}=(1,0, \ldots, 0)^{\top}$, i.e. the initial state corresponds to the "restart state". When the number $m$ of subintervals is sufficiently large (say $m \geq 200$ ), this approach provides an effective method that allows the ARL and SDRL of continuous statistics to be accurately evaluated by using the following classical formulas from the theory of Markov chains (see, for instance Neuts ${ }^{30}$ or Latouche and Ramaswami ${ }^{31}$ )

$$
\begin{aligned}
\mathrm{ARL} & =\mathbf{q}^{\boldsymbol{\top}}(\mathbf{I}-\mathbf{Q})^{-1} \mathbf{1}, \\
\mathrm{SDRL} & =\sqrt{2 \mathbf{q}^{\boldsymbol{\top}}(\mathbf{I}-\mathbf{Q})^{-2} \mathbf{Q} \mathbf{1}+\mathrm{ARL}(1-\mathrm{ARL})} .
\end{aligned}
$$

Using the "standard" Markov Chain method presented above, Figure 1 shows several plots of $\mathrm{ARL}_{0}$ (plain lines) in function of the number of sub-intervals $m \in$ $\{100,110, \ldots, 500\}$ used in the Markov chain for the upper-sided WSR EWMA chart with parameters $(\lambda=0.2, K=2.7)$ and $n \in\{5,10,15,20\}$. In addition, $\mathrm{ARL}_{0}$ values obtained using $10^{5}$ Monte Carlo simulation runs are also depicted with dashed lines. From these plots, it can clearly be concluded that the $\mathrm{ARL}_{0}$ values of the upper-sided WSR EWMA chart obtained using the "standard" 
Markov Chain method of Brook and Evans ${ }^{21}$ heavily fluctuate depending on the value of $m$ and they do not exhibit any obvious monotonic convergence when the number of sub-intervals $m$ increases. In general, as the number of subintervals $m$ increases, the results tend to be more "steady", but still, as it can be seen, even for $m \approx 500$, there are cases where the results differ significantly from the ones obtained using simulations. An immediate consequence of these results is that it is almost impossible to "optimize" (i.e. find optimal pairs $(\lambda, K))$ the upper-sided WSR EWMA chart if $\mathrm{ARL}_{0}$ values are computed using the standard Markov Chain method of Brook and Evans ${ }^{21}$. Therefore, a more efficient technique is needed for the exact and robust determination of ARL values regardless the values of $m, n$ or the pair $(\lambda, K)$. In the following sections we will provide an efficient and simple approach in which the ARL values are no longer affected by the number of sub-interval $m$ and become almost stable even for $m<200$.

\subsection{The upper-sided C-WSR EWMA control chart}

As it has been illustrated in the previous section, ARL values are highly affected by the number of sub-intervals $m$. For this reason, Wu et al. ${ }^{26}$ suggested to use a transformation (called "continuousify") of the discrete nonparametric statistic to be monitored in order to make the results, obtained by the traditional approach of Brook and Evans ${ }^{21}$, more robust. More specifically, they proposed a transformation of the discrete statistic as a mixture of Normal distributions (or kernels). In particular, suppose that $X_{t}, t=1,2, \ldots$ represents a sequence of i.i.d. discrete random variables, each of them defined on $\Psi=\left\{\psi_{1}, \psi_{2}, \ldots\right\}$ with corresponding p.m.f. function $f_{X}(\psi \mid \boldsymbol{\theta})$ where $\boldsymbol{\theta}$ represents a vector of parameters. Then, according to $\mathrm{Wu}$ et al. ${ }^{26}$ a new continuous random variable denoted as $X_{t}^{*}$ can be defined as a mixture of normally distributed random variables $Y_{t}^{*}$ where, for each $\psi_{t} \in \Psi, Y_{t}^{*} \sim N\left(\psi_{t}, \sigma\right)$. Then, the corresponding p.m.f. $f_{X^{*}}(x \mid \boldsymbol{\theta})$ and c.d.f. $F_{X^{*}}(x \mid \boldsymbol{\theta})$ of $X_{t}^{*}$ will be computed as:

$$
\begin{aligned}
f_{X^{*}}(x \mid \boldsymbol{\theta}) & =\sum_{\psi \in \Psi} f_{X}(\psi \mid \boldsymbol{\theta}) f_{\mathrm{N}}(x \mid \psi, \sigma), \\
F_{X^{*}}(x \mid \boldsymbol{\theta}) & =\sum_{\psi \in \Psi} f_{X}(\psi \mid \boldsymbol{\theta}) F_{\mathrm{N}}(x \mid \psi, \sigma),
\end{aligned}
$$

where $f_{\mathrm{N}}(x \mid \psi, \sigma)$ and $F_{\mathrm{N}}(x \mid \psi, \sigma)$ are the p.d.f. and c.d.f. of the Normal $(\psi, \sigma)$ distribution, respectively, in which $\sigma>0$ is the so-called "continuousify" parameter to be fixed (which has nothing to deal with the original distribution of X). Therefore, for our proposed scheme based on the Wilcoxon Signed Rank statistic, since the domain in which $\mathrm{SR}_{t}$ is defined is $\Psi=\left\{-\frac{n(n+1)}{2},-\frac{n(n+1)}{2}+\right.$ $\left.2, \ldots, \frac{n(n+1)}{2}-2, \frac{n(n+1)}{2}\right\}$, we suggest to transform the statistic $\mathrm{SR}_{t}$ into a new continuous one denoted as $\mathrm{SR}_{t}^{*}$ with corresponding p.d.f. $f_{\mathrm{SR}_{t}^{*}}\left(s \mid n, p_{1}\right)$ and c.d.f. $F_{\mathrm{SR}_{t}^{*}}\left(s \mid n, p_{1}\right)$ defined for $s \in \Psi$ as:

$$
\begin{aligned}
f_{\mathrm{SR}_{t}^{*}}\left(s \mid n, p_{1}\right) & =\sum_{\psi \in \Psi} f_{\mathrm{SR}_{t}^{+}}\left(\frac{\psi+\frac{n(n+1)}{2}}{2} \mid n, p_{1}\right) f_{\mathrm{N}}(s \mid \psi, \sigma), \\
F_{\mathrm{SR}_{t}^{*}}\left(s \mid n, p_{1}\right) & =\sum_{\psi \in \Psi} f_{\mathrm{SR}_{t}^{+}}\left(\frac{\psi+\frac{n(n+1)}{2}}{2} \mid n, p_{1}\right) F_{N}(s \mid \psi, \sigma),
\end{aligned}
$$


where $f_{\mathrm{SR}_{t}^{+}}\left(\ldots \mid n, p_{1}\right)$ is the p.m.f. of the $\mathrm{SR}_{t}^{+}$statistic as defined in Section 2.1. Therefore, the charting statistic of the "continuousified" one-sided WSR EWMA (denoted as C-WSR EWMA chart) will be defined as:

$$
Z_{t}^{*}=\max \left(0, \lambda \mathrm{SR}_{t}^{*}+(1-\lambda) Z_{t-1}^{*}\right), Z_{0}^{*}=0,
$$

with fixed asymptotic control limits:

$$
\mathrm{UCL}=\mathrm{E}\left(\mathrm{SR}_{t}^{*}\right)+K \sqrt{\mathrm{V}\left(\mathrm{SR}_{t}^{*}\right)} \times \sqrt{\frac{\lambda}{2-\lambda}} .
$$

For the computation of the mean $\mathrm{E}\left(\mathrm{SR}_{t}^{*}\right)$ and variance $\mathrm{V}\left(\mathrm{SR}_{t}^{*}\right)$ of $\mathrm{SR}_{t}^{*}$ it can be easily proved that (see Appendix):

$$
\begin{aligned}
\mathrm{E}\left(\mathrm{SR}_{t}^{*}\right) & =\mathrm{E}\left(\mathrm{SR}_{t}\right), \\
\mathrm{V}\left(\mathrm{SR}_{t}^{*}\right) & =\mathrm{V}\left(\mathrm{SR}_{t}\right)+\sigma^{2} .
\end{aligned}
$$

In order to obtain the RL properties of the upper-sided C-WSR EWMA control chart, the standard discrete-time Markov chain approach of Brook and Evans ${ }^{21}$ presented in Section 2 will be used with the only difference that the p.m.f. of $\mathrm{SR}_{t}$ will be replaced by the p.m.f. of $\mathrm{SR}_{t}^{*}$ in the computation of the transient probabilities $Q_{k, i}$.

For a better understanding of the design of the C-WSR EWMA chart a brief example is presented in Table 1. The first column of this table represents the sample number, $t=\{1,2, \ldots, 10\}$, the second column contains the observed (simulated) values $\mathrm{SR}_{t}$ for a sample size $n=10$ and $p_{0}=0.5$, while the third column contains the corresponding transformed $\mathrm{SR}_{t}^{*}$. Finally, the corresponding values of the charting statistics $Z_{t}^{*}$ are presented in the rightmost column. For illustrative purposes the following parameters have been fixed: $\lambda=0.2$ (smoothing parameter), $\sigma=0.2$ ("continuousify" parameter) and $Z_{0}^{*}=0$ (no head-start feature). More specifically:

- For $t=1$ we have $\mathrm{SR}_{1}=-11$. The corresponding value for $\mathrm{SR}_{1}^{*}$ is computed by generating a $\mathrm{N}(-11,0.2)$ random variable. The value of the charting statistic is $Z_{1}^{*}=\max (0,0.2 \times(-10.6823)+0.8 \times 0)=\max (0,-2.1365)=$ 0 .

- For $t=2$ we have $\mathrm{SR}_{2}=21$. The corresponding value for $\mathrm{SR}_{2}^{*}$ is computed by generating a $\mathrm{N}(21,0.2)$ random variable. The value of the charting statistic is $Z_{2}^{*}=\max (0,0.2 \times 20.9128+0.8 \times 0)=\max (0,4.1826)=4.1826$.

- For $t=10$ we have $\mathrm{SR}_{10}=5$. The corresponding value for $\mathrm{SR}_{10}^{*}$ is computed by generating a $\mathrm{N}(5,0.2)$ random variable. The value of the charting statistic is $Z_{10}^{*}=\max (0,0.2 \times 5.2996+0.8 \times 5.2678)=\max (0,5.2742)=$ 5.2742 .

It should be pointed out that even though the operations of this chart requires random numbers to be generated, its Run Length properties (ARL, SDRL, ...), are obtained directly through the distribution of the $\mathrm{SR}_{t}^{*}$ with the exact Markov 
chain method shown above without the need of perfoming any simulations. This fact has also mentioned by Wu et al. ${ }^{26}$.

In order to show the efficiency of the "continuousify" method, Table 2 presents ARL values of the WSR EWMA (without "continuousify") and C-WSR EWMA (with "continuousify") charts for $\lambda=0.2, K=2.7$ and for several combinations of $\left(n, p_{1}\right)$. In Table 2 , the value $\sigma=0.2$ has been fixed but, as it will be highligthed in the next Section, the results are are not significantly affected by this choice. Based on the results in Table 2 we draw the following conclusions:

- as this has been already shown in Figure 1 (but only for the in-control case), the ARL values obtained without "continuousify" (i.e. the WSR EWMA chart) strongly fluctuate depending on the value of $m$. Clearly, they do not exhibit any monotonic convergence when the number of subintervals $m$ increases. For instance, in the case $\left(n, p_{1}\right)=(7,0.53)$, the ARL values obtained without "continuousify" fluctuate from 112 to 354.4.

- on the contrary, for $m \geq 100$, the ARL values obtained with the "continuousify" method (i.e. the C-WSR EWMA chart) exhibit a strong stability and they seem to converge rapidly to a reliable value. Even for $m=100$ the results obtained with the "continuousify" approach are very reliable. For instance, using the same case $\left(n, p_{1}\right)=(7,0.53)$, the ARL values obtained with "continuousify" converge rapidly to 150.4.

We have also computed the ARL values of the WSR EWMA chart using $10^{6}$ Monte-Carlo simulation runs (see bottom of Table 2). What can be seen is that the out-of-control ARL values obtained with the "continuousify" method (see for example the case $m=400$ ), i.e. 150.4, 28.4, 109.2, 328.0 are almost the same or just a bit larger to the ones obtained using simulations, i.e. 150.4, 28.3, 109.0, 326.7 .

\section{$3 \quad$ Numerical analysis}

In this Section we will investigate the impact of the choice of i) the parameter $\sigma$ and ii) the kernel used in the "continuousify" method (for the moment, only the normal kernel has been considered), on the ARL of the C-WSR EWMA chart. We will also present optimal design parameters for this control chart.

\subsection{Effect of $\sigma$}

In Table 3 , setting $\lambda=0.2$ and $K=2.7$, we present the ARL values under several scenarios for fixed values of $\sigma=\{0.1,0.15, \ldots, 0.25\}$ and different combinations of $\left(n, p_{1}\right)$. It is clear from these results that regardless the value of $\sigma \in[0.1,0.25]$, the ARL values obtained for the C-WSR EWMA chart are i) very stable, even for small values of $m \approx 100$ and ii) not seriously affected by the choice of $\sigma$ with some tiny differences occuring in the first point. As a consequence, as long as $\sigma$ is neither too small nor too large, the results are not affected. Therefore we suggest to set $\sigma=0.2$ as a reasonable choice for the value of the "continuousify" parameter. 


\subsection{Effect of the kernel}

In this paper (as in Wu et al. ${ }^{26}$ ), the distribution / kernel used for transforming the discrete random variable $\mathrm{SR}_{t}$ into a continuous one denoted as $\mathrm{SR}_{t}^{*}$ has been chosen to be the normal $(\psi, \sigma)$ distribution (see (2) and (3)) which can be simply derived from the $\mathrm{N}(0,1)$ distribution by a straightforward standardization. A legitimate question is what happens to the previous results concerning the $\mathrm{C}$ WSR EWMA chart if the normal kernel used in (2) and (3) is replaced by another continuous one? Are the ARL values obtained by this modification different from what has been obtained with the normal kernel? Therefore, the goal is to investigate the impact of the choice of the kernel on the ARL values of the C-WSR EWMA chart if (2) and (3) are replaced by

$$
\begin{aligned}
f_{\mathrm{SR}_{t}^{*}}\left(s \mid n, p_{1}\right) & =\sum_{\psi \in \Psi} f_{\mathrm{SR}_{t}^{+}}\left(\frac{\psi+\frac{n(n+1)}{2}}{2} \mid n, p_{1}\right) K\left(\frac{s-\psi}{\sigma}\right) \\
F_{\mathrm{SR}_{t}^{*}}\left(s \mid n, p_{1}\right) & =\sum_{\psi \in \Psi} f_{\mathrm{SR}_{t}^{+}}\left(\frac{\psi+\frac{n(n+1)}{2}}{2} \mid n, p_{1}\right) K\left(\frac{s-\psi}{\sigma}\right),
\end{aligned}
$$

where $K(\ldots)$ is a standardized continuous kernel as the ones listed in Table 4. In Table 5, ARL values of the C-WSR EWMA chart are presented for parameters $\lambda=0.2, K=2.7$, for $\sigma=0.2, n \in\{7,13,15,18,20,25\}$ and for the kernels listed in Table 4. As it can be noted, for a fixed value of $n$, the choice of the kernel clearly seems to have almost no impact on the results, no matter the value of $m$. This implies that the user is totally free to use the kernel of his/her choice without having to worry much about the reliability of the result.

\subsection{Optimal Design parameters for the C-WSR EWMA chart}

In this section, we present the results of a numerical study for the performance of the C-WSR EWMA control chart. The desired in-control ARL value $\left(\mathrm{ARL}_{0}\right)$ is set equal to 370 and no head-start feature has been used $\left(Z_{0}^{*}=0\right)$. For the computations, we used the Markov chain method presented in Section 2 and all the calculations were performed in $\mathrm{R}$. In Table 6 , we give the optimal design parameters $\left(\lambda^{*}, K^{*}\right)$ for different shifts $(p)$ and sample sizes $(n)$ along with the corresponding $\mathrm{ARL}_{1}$ values setting the number of subintervals equal to $m=200$. For the determination of the optimal pair $\left(\lambda^{*}, K^{*}\right)$ for the C-WSR EWMA chart, we suggest the following procedure: Find out the optimal pair $\left(\lambda^{*}, K^{*}\right)$ such that for fixed value of $n$, we have $\operatorname{ARL}\left(n, \lambda^{*}, K^{*}, p=0.5\right)=370$ and, for a fixed value of $p, \operatorname{ARL}\left(n, \lambda^{*}, K^{*}, p\right)$ is the smallest out-of-control ARL. Note that $p$ is the magnitude of the shift. If $p=0.5$ the process is in-control and, when $p$ is larger than 0.5 , the process is out-of-control.

The pairs are given in Table 6 for various combinations of $n=\{5,6,7, \ldots, 20\}$ and $p=\{0.55,0.60,0.65, \ldots, 0.95\}$. In each cell, the three numbers given are $\lambda^{*}, K^{*}$ and $\mathrm{ARL}_{1}$. Apparently, these values can be used to design the chart, if the practitioner knows at least on the average the out-of-control value $p$. For example, if $n=10$ and $p=0.6$ the proper parameters are $\lambda^{*}=0.07$ and $K^{*}=2.523$. These values give $\mathrm{ARL}_{1}=20.6$. 


\section{An illustrative example}

In this Section an illustrative example is provided to show a practical Phase II implementation of the design and operation of our proposed chart. This example was originally discussed by Celano et al. ${ }^{32}$ in which the quality characteristic to be monitored is the radial error, defined as "a quality characteristic frequently monitored in hole drilling processes of mechanical parts and assembly processes of printed circuit boards". At each sampling point $t$, a subgroup of size $n=20$ is collected in order to detect a shift in the median of the quality of interest such that $p_{0}=0.5$ shifts to $p_{1}=0.7$. As shown in Table 6 , the optimal parameters to be used are $\lambda=0.34$ and $K=2.785$. In addition, as shown in Celano et al. ${ }^{32}$ the in-control value of the median for the radial error is $\theta_{0}=0.338$. In Table 7 the values of the simulated radial errors $X_{t, j}$ for $t \in\{1,2 \ldots, 10\}$ and $j \in\{1,2 \ldots, 20\}$ are provided and the values of $\mathrm{SR}_{t}, \mathrm{SR}_{t}^{*}$, and $Z_{t}^{*}$ are also reported. In Figure 2 the differences $X_{t, j}-\theta_{0}$ for $t \in\{1,2 \ldots, 10\}$ are plotted, where at each sampling point, $t$, more than one values correspond to ties between the differences of $X_{t, j}$ and $\theta_{0}$. The values of the charting statistic $Z_{t}^{*}$ are plotted in Figure 3. It can be seen that at the $4^{t h}$ sampling point $(t=4)$ an out-of-control signal is given stating that the process median has changed.

\section{Conclusions}

In this paper we proposed a modified distribution-free EWMA control chart based on the Wilcoxon signed rank statistic called as the C-WSR EWMA chart. We aimed to present a robust technique which guarantees steady results for its Run Length properties. Using the "continuousify" method introduced by Wu et al. ${ }^{26}$ we determined its RL properties showing that the number of cutpoints does not affect the results. Additionally, we tested the efficiency of this method under the use of several kernels besides the Gaussian and we saw that no significant differences exist. It should be noted that our work was mainly focused on providing an enhanced method for the determination of the chart's RL properties rather than examining its superiority versus other schemes. It is worth stretching that, its in- and out-of-control performances were derived regardless the process underlying distribution for monitoring any percentile of interest.

As a future work many things can be pursued. For instance, the "continuousify" method could be applied in EWMA-type schemes where other nonparametric statistics are considered such as the Mann-Whitney, and the Ansari-Bradley statistics. Additionally, it would be interesting to examine the performance of the C-WSR EWMA chart in the presence of ties in the population. Finally, it would be challenging to investigate the use of similar kernel-based techniques in distribution-free EWMA schemes designed for monitoring bivariate processes.

\section{Appendix}

Let as denote $\mathrm{E}_{N}(X)=\mu$ and $\mathrm{V}_{N}(X)=\sigma^{2}$ as the mean and variance of a random variable, $\mathrm{X}$, from a Normal distribution. For the computation of the mean of $\mathrm{SR}_{t}^{*}$ we have: 


$$
\begin{aligned}
\mathrm{E}\left(\mathrm{SR}_{t}^{*}\right) & =\int_{-\infty}^{\infty} s \times f_{\mathrm{SR}_{t}^{*}}\left(s \mid n, p_{1}\right) d s \\
& =\int_{-\infty}^{\infty} s \times \sum_{\psi \in \Psi} f_{\mathrm{SR}_{t}^{+}}\left(\frac{\psi+\frac{n(n+1)}{2}}{2} \mid n, p_{1}\right) \times f_{\mathrm{N}}(s \mid \psi, \sigma) d s \\
& =\sum_{\psi \in \Psi}\left[f_{\mathrm{SR}_{t}^{+}}\left(\frac{\left.\left.\psi+\frac{n(n+1)}{2} \mid n, p_{1}\right) \times \int_{-\infty}^{\infty} s \times f_{\mathrm{N}}(s \mid \psi, \sigma) d s\right]}{2} \mid n, p_{1}\right) \times \mathrm{E}_{N}(s)\right] \\
& =\sum_{\psi \in \Psi}\left[f_{\mathrm{SR}_{t}^{+}}\left(\frac{\psi+\frac{n(n+1)}{2}}{2} \mid n, p_{1}\right) \times \psi\right] \\
& =\sum_{\psi \in \Psi}\left[f _ { \mathrm { SR } _ { t } ^ { + } } \left(\frac{\psi+\frac{n(n+1)}{2}}{2} \mid\right.\right. \\
& =\mathrm{E}\left(\mathrm{SR}_{t}\right)
\end{aligned}
$$

Similarly, using the fact that $\mathrm{E}\left(\mathrm{SR}_{t}^{*}\right)=\mathrm{E}\left(\mathrm{SR}_{t}\right)$ the variance of $\mathrm{SR}_{t}^{*}$ is computed as:

$$
\begin{aligned}
& \mathrm{V}\left(\mathrm{SR}_{t}^{*}\right)=\mathrm{E}\left(\mathrm{SR}_{t}^{*}\right)^{2}-\left(\mathrm{E}_{\left.\left(\mathrm{SR}_{t}^{*}\right)\right)^{2}}\right. \\
& =\int_{-\infty}^{\infty} s^{2} \times f_{\mathrm{SR}_{t}^{*}}\left(s \mid n, p_{1}\right) d s-\left(\mathrm{E}_{\left.\left(\mathrm{SR}_{t}^{*}\right)\right)^{2}}\right. \\
& =\int_{-\infty}^{\infty} s^{2} \times \sum_{\psi \in \Psi}\left[f_{\mathrm{SR}_{t}^{+}}\left(\frac{\psi+\frac{n(n+1)}{2}}{2} \mid n, p_{1}\right) \times f_{\mathrm{N}}(s \mid \psi, \sigma)\right] d s-\left(\mathrm{E}_{\left.\left(\mathrm{SR}_{t}\right)\right)^{2}}\right. \\
& =\sum_{\psi \in \Psi}\left[f_{\mathrm{SR}_{t}^{+}}\left(\frac{\psi+\frac{n(n+1)}{2}}{2} \mid n, p_{1}\right) \times \int_{-\infty}^{\infty} s^{2} \times f_{\mathrm{N}}(s \mid \psi, \sigma) d s\right]-\left(\mathrm{E}_{\left.\left(\mathrm{SR}_{t}\right)\right)^{2}}\right. \\
& =\sum_{\psi \in \Psi}\left[f_{\mathrm{SR}_{t}^{+}}\left(\frac{\psi+\frac{n(n+1)}{2}}{2} \mid n, p_{1}\right) \times \mathrm{E}_{N}\left(s^{2}\right)\right]-\left(\mathrm{E}\left(\mathrm{SR}_{t}\right)\right)^{2} \\
& =\sum_{\psi \in \Psi}\left[f_{\mathrm{SR}_{t}^{+}}\left(\frac{\psi+\frac{n(n+1)}{2}}{2} \mid n, p_{1}\right) \times\left(\mathrm{V}_{N}(s)+\left(\mathrm{E}_{N}(s)\right)^{2}\right)\right]-\left(\mathrm{E}\left(\mathrm{SR}_{t}\right)\right)^{2} \\
& =\sum_{\psi \in \Psi}\left[f_{\mathrm{SR}_{t}^{+}}\left(\frac{\psi+\frac{n(n+1)}{2}}{2} \mid n, p_{1}\right) \times\left(\sigma^{2}+\psi^{2}\right)\right]-\left(\mathrm{E}_{\left.\left(\mathrm{SR}_{t}\right)\right)^{2}}\right. \\
& =\sigma^{2} \times \sum_{\psi \in \Psi} f_{\mathrm{SR}_{t}^{+}}\left(\frac{\psi+\frac{n(n+1)}{2}}{2} \mid n, p_{1}\right)+\sum_{\psi \in \Psi} \psi^{2} \times f_{\mathrm{SR}_{t}^{+}}\left(\frac{\psi+\frac{n(n+1)}{2}}{2} \mid n, p_{1}\right)-\left(\mathrm{E}\left(\mathrm{SR}_{t}\right)\right)^{2} \\
& =\sigma^{2}+\mathrm{E}\left(\mathrm{SR}_{t}\right)^{2}-\left(\mathrm{E}\left(\mathrm{SR}_{t}\right)\right)^{2} \\
& =\sigma^{2}+\mathrm{V}\left(\mathrm{SR}_{t}\right)
\end{aligned}
$$




\section{References}

[1] W.A. Shewhart. Statistical Method from the Viewpoint of quality control, Graduate School of Agriculture. Washington, DC, 1939.

[2] SW Roberts. Control chart tests based on geometric moving averages. Technometrics, 1:239-250, 1959.

[3] E.S. Page. Continuous Inspection Schemes. Biometrika, 41(1/2):100-115, 1954.

[4] S. Chakraborti, P. Van der Laan, and S.T. Bakir. Nonparametric Control Charts: an Overview and Some Results. Journal of Quality Technology, 33 (3):304-315, 2001.

[5] Peihua Qiu. Some perspectives on nonparametric statistical process control. Journal of Quality Technology, 50(1):49-65, 2018.

[6] S. Chakraborti and M.A. Graham. Nonparametric (Distribution-Free) Control Charts: An Updated Overview and Some Results. Quality Engineering, 31(4):523-544, 2019.

[7] R.W. Amin and A.J. Searcy. A Nonparametric Exponentially Weighted Moving Average Control Scheme. Communications in Statistics-Simulation and Computation, 20(4):1049-1072, 1991.

[8] M.A. Graham, S. Chakraborti, and S.W. Human. A Nonparametric EWMA Sign Chart for Location Based on Individual Measurements. Quality Engineering, 23(3):227-241, 2011.

[9] S.-F. Yang, J.-S. Lin, and S.W. Cheng. A New Nonparametric EWMA Sign Control Chart. Expert Systems with Applications, 38(5):6239-6243, 2011.

[10] M. Aslam, M. Azam, and C.H. Jun. A New Exponentially Weighted Moving Average Sign Chart using Repetitive Sampling. Journal of Process Control, 24(7):1149-1153, 2014.

[11] M. Riaz. A Sensitive Non-Parametric EWMA Control Chart. Journal of the Chinese Institute of Engineers, 38(2):208-219, 2015.

[12] S.L. Lu. An Extended Nonparametric Exponentially Weighted Moving Average Sign Control Chart. Quality and Reliability Engineering International, 31(1):3-13, 2015.

[13] A. Haq. A New Nonparametric Synthetic EWMA Control Chart for Monitoring Process Mean. Communications in Statistics-Simulation and Computation, 48(6):1665-1676, 2019.

[14] S.Y. Li, L.C. Tang, and S.H. Ng. Nonparametric CUSUM and EWMA Control Charts for Detecting Mean Shifts. Journal of Quality Technology, 42(2):209-226, 2010.

[15] M.A. Graham, S. Chakraborti, and S.W. Human. A Nonparametric Exponentially Weighted Moving Average Signed-Rank Chart for Monitoring Location. Computational Statistics \& Data Analysis, 55(8):2490-2503, 2011. 
[16] N. Chakraborty, S. Chakraborti, S.W. Human, and N. Balakrishnan. A Generally Weighted Moving Average Signed-Sank Control Chart. Quality and Reliability Engineering International, 32(8):2835-2845, 2016.

[17] H.Z. Abid, M.and Nazir, M. Riaz, and Z. Lin. An Efficient Nonparametric EWMA Wilcoxon Signed-Rank Chart for Monitoring Location. Quality and Reliability Engineering International, 33(3):669-685, 2017.

[18] Muhammad Ali Raza, Tahir Nawaz, Muhammad Aslam, Sajjad Haider Bhatti, and Rehan Ahmed Khan Sherwani. A New Nonparametric Double Exponentially Weighted Moving Average Control Chart. Quality and Reliability Engineering International, 36(1):68-87, 2020.

[19] Vasileios Alevizakos, Christos Koukouvinos, and Kashinath Chatterjee. A Nonparametric Double Generally Weighted Moving Average Signed-Rank Control Chart for Monitoring Process Location. Quality and Reliability Engineering International, 2020.

[20] K Mabude, JC Malela-Majika, P Castagliola, and SC Shongwe. Generally Weighted Moving Average Monitoring Schemes-Overview and Perspectives.

[21] D. Brook and D.A. Evans. An Approach to the Probability Distribution of CUSUM Run Length. Biometrika, 59(3):539-549, 1972.

[22] C.H. Weiß. EWMA Monitoring of Correlated Processes of Poisson Counts. Quality Technology $\& 3$ Quantitative Management, 6(2):137-153, 2009.

[23] P. Castagliola, K.P. Tran, G. Celano, A.C. Rakitzis, and P.E. Maravelakis. An EWMA-Type Sign Chart With Exact Run Length Properties. Journal of Quality Technology, 51(1):51-63, 2019.

[24] A.C. Rakitzis, P. Castagliola, and P.E. Maravelakis. A New Memory-type Monitoring Technique for Count Data. Computers $\& 3$ Industrial Engineering, 85:235-247, 2015.

[25] A. Tang, J. Sun, X. Hu, and P. Castagliola. A New Nonparametric Adaptive EWMA Control Chart with Exact Run Length Properties. Computers \& Industrial Engineering, 130:404-419, 2019.

[26] S. Wu, P. Castagliola, and G. Celano. A Distribution-free EWMA Control Chart for Monitoring Time-Between-Events-and-Amplitude Data. Journal of Applied Statistics, pages 1-21, 2020.

[27] Jean Dickinson Gibbons and Subhabrata Chakraborti. Nonparametric Statistical Inference: Revised and Expanded. CRC press, 2014.

[28] R.L. McCornack. Extended Tables of the Wilcoxon Matched Pair Signed Rank Statistic. Journal of the American Statistical Association, 60(311): 864-871, 1965.

[29] B.M. Bennett. On the Non-Null Distribution of Wilcoxon's Signed Rank Test. Metrika, 19(1):36-38, 1972. 
[30] M.F. Neuts. Matrix-Geometric Solutions in Stochastic Models: an Algorithmic Approach. Dover Publications Inc, New York, 1981.

[31] G. Latouche and V. Ramaswami. Introduction to Matrix Analytic Methods in Stochastic Modeling. ASA-SIAM, Philadelphia, 1999.

[32] G. Celano, P. Castagliola, S. Chakraborti, and G. Nenes. On the Implementation of the Shewhart Sign Control Chart for Low-volume Production. International Journal of Production Research, 54(19):5886-5900, 2016.

Table 1: Implementation Example Data

\begin{tabular}{cccc}
\hline$t$ & $\mathrm{SR}_{t}$ & $\mathrm{SR}_{t}^{*}$ & $Z_{t}^{*}$ \\
\hline 1 & -11 & -10.6823 & 0 \\
2 & 21 & 20.9128 & 4.1826 \\
3 & 27 & 26.5635 & 8.6587 \\
4 & -9 & -9.2340 & 5.0802 \\
5 & 1 & 0.9568 & 4.2555 \\
6 & -21 & -21.0686 & 0 \\
7 & 3 & 2.9014 & 0.5803 \\
8 & 17 & 17.1334 & 3.8909 \\
9 & 11 & 10.7754 & 5.2678 \\
10 & 5 & 5.2996 & 5.2742 \\
\hline
\end{tabular}


Table 2: Comparison of out-of-control ARL values for the WSR EWMA (without "continuousify") and C-WSR EWMA (with "continuousify" and $\sigma=0.2$ ) chart when $\lambda=0.2$ and $K=2.7$

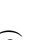

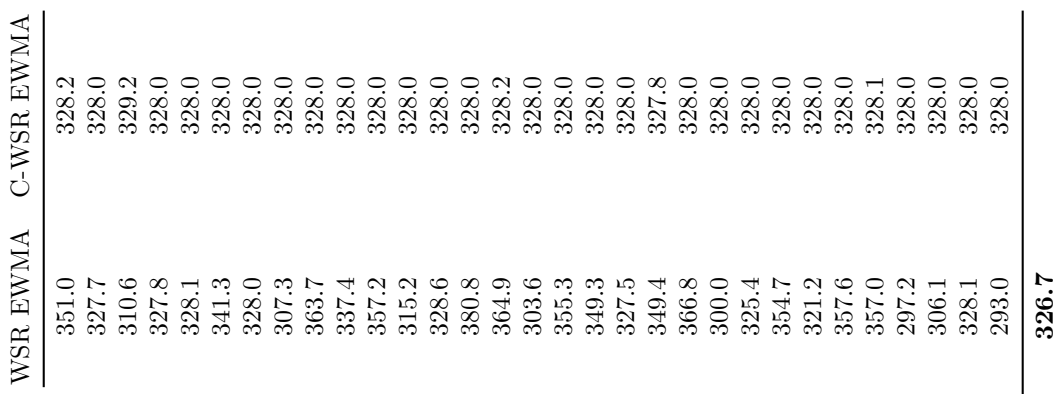

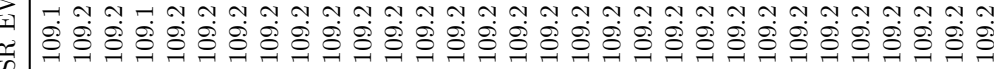

กิ?

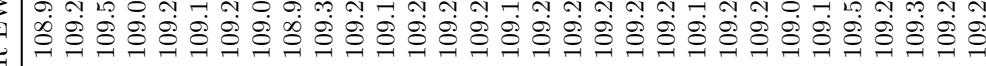

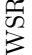

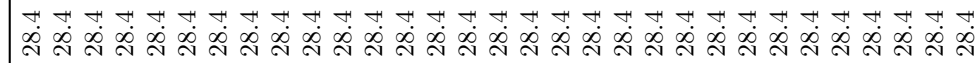

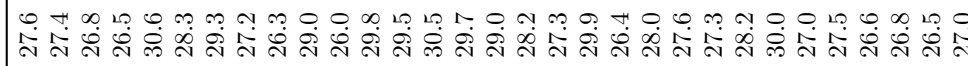

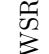

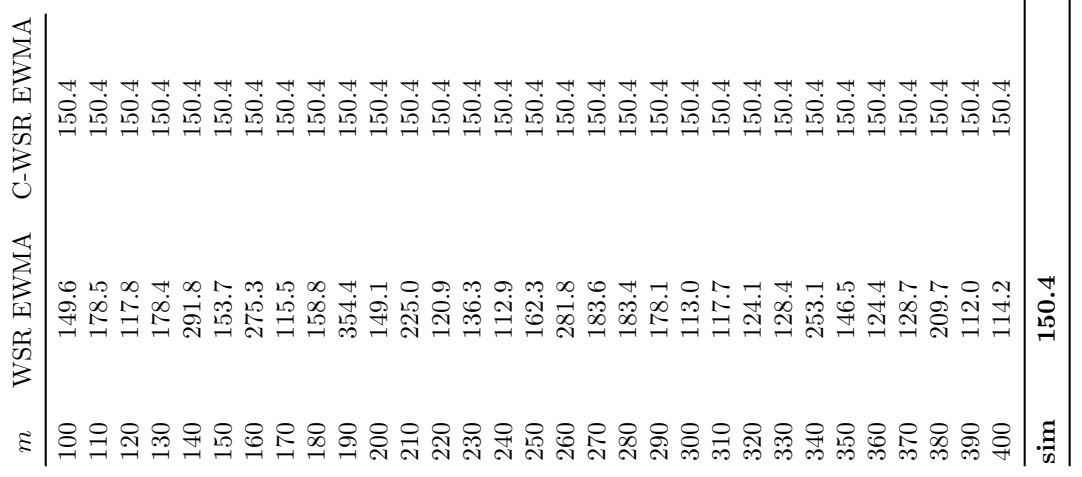


Table 3: ARL values of the C-WSR EWMA chart for $\lambda=0.2, K=2.7$ and for fixed values of $\sigma=\{0.1,0.15, \ldots, 0.25\}$ and different combinations of $\left(n, p_{1}\right)$

\begin{tabular}{ccccc}
\hline \multicolumn{5}{c}{$\left(n, p_{1}\right)=(5,0.5)$} \\
$m$ & 0.1 & 0.15 & 0.2 & 0.25 \\
\hline 100 & 388.8 & 388.6 & 388.6 & 388.5 \\
110 & 388.8 & 388.6 & 388.6 & 388.5 \\
120 & 388.7 & 388.6 & 388.6 & 388.5 \\
130 & 388.7 & 388.7 & 388.6 & 388.5 \\
140 & 388.7 & 388.7 & 388.6 & 388.6 \\
150 & 388.7 & 388.7 & 388.6 & 388.6 \\
160 & 388.7 & 388.7 & 388.6 & 388.6 \\
170 & 388.7 & 388.7 & 388.7 & 388.6 \\
180 & 388.7 & 388.7 & 388.7 & 388.6 \\
190 & 388.7 & 388.7 & 388.7 & 388.6 \\
200 & 388.8 & 388.7 & 388.7 & 388.6 \\
210 & 388.8 & 388.7 & 388.7 & 388.6 \\
220 & 388.8 & 388.7 & 388.7 & 388.6 \\
230 & 388.8 & 388.7 & 388.7 & 388.6 \\
240 & 388.8 & 388.7 & 388.7 & 388.6 \\
250 & 388.8 & 388.7 & 388.7 & 388.6 \\
260 & 388.8 & 388.7 & 388.7 & 388.6 \\
270 & 388.8 & 388.7 & 388.7 & 388.6 \\
280 & 388.8 & 388.7 & 388.7 & 388.6 \\
290 & 388.8 & 388.7 & 388.7 & 388.6 \\
300 & 388.8 & 388.7 & 388.7 & 388.6 \\
310 & 388.8 & 388.8 & 388.7 & 388.6 \\
320 & 388.8 & 388.8 & 388.7 & 388.6 \\
330 & 388.8 & 388.8 & 388.7 & 388.6 \\
340 & 388.8 & 388.8 & 388.7 & 388.6 \\
350 & 388.8 & 388.8 & 388.7 & 388.6 \\
360 & 388.8 & 388.8 & 388.7 & 388.6 \\
370 & 388.8 & 388.8 & 388.7 & 388.6 \\
380 & 388.8 & 388.8 & 388.7 & 388.6 \\
390 & 388.8 & 388.8 & 388.7 & 388.6 \\
400 & 388.8 & 388.8 & 388.7 & 388.6 \\
\hline & & & &
\end{tabular}

\begin{tabular}{ccccc}
\hline \multicolumn{5}{c}{$\left(n, p_{1}\right)=(10,0.5)$} \\
$m$ & 0.1 & 0.15 & 0.2 & 0.25 \\
\hline 100 & 346.0 & 346.0 & 346.1 & 346.1 \\
110 & 346.2 & 346.2 & 346.2 & 346.1 \\
120 & 346.3 & 346.2 & 346.2 & 346.2 \\
130 & 346.0 & 346.1 & 346.1 & 346.2 \\
140 & 347.4 & 346.6 & 346.3 & 346.2 \\
150 & 346.2 & 346.2 & 346.2 & 346.2 \\
160 & 346.2 & 346.2 & 346.2 & 346.2 \\
170 & 346.2 & 346.2 & 346.2 & 346.2 \\
180 & 346.1 & 346.2 & 346.2 & 346.2 \\
190 & 346.2 & 346.2 & 346.2 & 346.2 \\
200 & 346.2 & 346.2 & 346.2 & 346.2 \\
210 & 346.2 & 346.2 & 346.2 & 346.2 \\
220 & 345.9 & 346.2 & 346.2 & 346.2 \\
230 & 346.3 & 346.2 & 346.2 & 346.2 \\
240 & 346.2 & 346.2 & 346.2 & 346.2 \\
250 & 346.3 & 346.2 & 346.2 & 346.2 \\
260 & 346.3 & 346.2 & 346.2 & 346.2 \\
270 & 346.2 & 346.2 & 346.2 & 346.2 \\
280 & 346.2 & 346.2 & 346.2 & 346.2 \\
290 & 346.2 & 346.2 & 346.2 & 346.2 \\
300 & 346.2 & 346.2 & 346.2 & 346.2 \\
310 & 346.3 & 346.3 & 346.2 & 346.2 \\
320 & 346.3 & 346.3 & 346.2 & 346.2 \\
330 & 346.3 & 346.3 & 346.2 & 346.2 \\
340 & 346.3 & 346.3 & 346.2 & 346.2 \\
350 & 346.3 & 346.3 & 346.3 & 346.2 \\
360 & 346.3 & 346.3 & 346.3 & 346.2 \\
370 & 346.3 & 346.3 & 346.3 & 346.3 \\
380 & 346.3 & 346.3 & 346.3 & 346.3 \\
390 & 346.3 & 346.3 & 346.3 & 346.3 \\
400 & 346.3 & 346.3 & 346.3 & 346.3 \\
\hline & & & &
\end{tabular}

\begin{tabular}{cccc}
\hline \multicolumn{4}{c}{$\left(n, p_{1}\right)=(7,0.55)$} \\
0.1 & 0.15 & 0.2 & 0.25 \\
\hline 89.7 & 89.7 & 89.8 & 89.8 \\
89.7 & 89.7 & 89.8 & 89.8 \\
89.7 & 89.8 & 89.8 & 89.8 \\
89.8 & 89.8 & 89.8 & 89.8 \\
89.8 & 89.8 & 89.8 & 89.8 \\
89.7 & 89.8 & 89.8 & 89.8 \\
89.7 & 89.8 & 89.8 & 89.8 \\
89.8 & 89.8 & 89.8 & 89.8 \\
89.8 & 89.8 & 89.8 & 89.8 \\
89.8 & 89.8 & 89.8 & 89.8 \\
89.8 & 89.8 & 89.8 & 89.8 \\
89.8 & 89.8 & 89.8 & 89.8 \\
89.8 & 89.8 & 89.8 & 89.8 \\
89.8 & 89.8 & 89.8 & 89.8 \\
89.8 & 89.8 & 89.8 & 89.8 \\
89.8 & 89.8 & 89.8 & 89.8 \\
89.8 & 89.8 & 89.8 & 89.8 \\
89.8 & 89.8 & 89.8 & 89.8 \\
89.8 & 89.8 & 89.8 & 89.8 \\
89.8 & 89.8 & 89.8 & 89.8 \\
89.8 & 89.8 & 89.8 & 89.8 \\
89.8 & 89.8 & 89.8 & 89.8 \\
89.8 & 89.8 & 89.8 & 89.8 \\
89.8 & 89.8 & 89.8 & 89.8 \\
89.8 & 89.8 & 89.8 & 89.8 \\
89.8 & 89.8 & 89.8 & 89.8 \\
89.8 & 89.8 & 89.8 & 89.8 \\
89.8 & 89.8 & 89.8 & 89.8 \\
89.8 & 89.8 & 89.8 & 89.8 \\
89.8 & 89.8 & 89.8 & 89.8 \\
89.8 & 89.8 & 89.8 & 89.8 \\
\hline \multicolumn{5}{c}{$\sigma$} & &
\end{tabular}

\begin{tabular}{cccc}
\hline \multicolumn{4}{c}{$\left(n, p_{1}\right)=(15,0.53)$} \\
0.1 & 0.15 & 0.2 & 0.25 \\
\hline 100.9 & 100.9 & 100.9 & 100.9 \\
100.9 & 100.9 & 100.9 & 100.9 \\
100.9 & 100.9 & 100.9 & 100.9 \\
100.8 & 100.8 & 100.9 & 100.9 \\
100.9 & 100.9 & 100.9 & 100.9 \\
100.9 & 100.9 & 100.9 & 100.9 \\
100.8 & 100.8 & 100.9 & 100.9 \\
100.9 & 100.9 & 100.9 & 100.9 \\
100.9 & 100.9 & 100.9 & 100.9 \\
101.2 & 101.9 & 101.9 & 100.9 \\
100.9 & 100.9 & 100.9 & 100.9 \\
100.9 & 100.9 & 100.9 & 100.9 \\
100.9 & 100.9 & 100.9 & 100.9 \\
100.9 & 100.9 & 100.9 & 100.9 \\
100.9 & 100.9 & 100.9 & 100.9 \\
100.9 & 100.9 & 100.9 & 100.9 \\
100.9 & 100.9 & 100.9 & 100.9 \\
100.9 & 100.9 & 100.9 & 100.9 \\
100.9 & 100.9 & 100.9 & 100.9 \\
100.9 & 100.9 & 100.9 & 100.9 \\
100.9 & 100.9 & 100.9 & 100.9 \\
100.9 & 100.9 & 100.9 & 100.9 \\
100.9 & 100.9 & 100.9 & 100.9 \\
100.9 & 100.9 & 100.9 & 100.9 \\
100.9 & 100.9 & 100.9 & 100.9 \\
100.9 & 100.9 & 100.9 & 100.9 \\
100.9 & 100.9 & 100.9 & 100.9 \\
100.9 & 100.9 & 100.9 & 100.9 \\
100.8 & 100.9 & 100.9 & 100.9 \\
100.9 & 100.9 & 100.9 & 100.9 \\
100.9 & 100.9 & 100.9 & 100.9 \\
\hline & & &
\end{tabular}

\begin{tabular}{cccc}
\hline \multicolumn{4}{c}{$\left(n, p_{1}\right)=(13,0.52)$} \\
0.1 & 0.15 & 0.2 & 0.25 \\
\hline 155.1 & 155.1 & 155.1 & 155.1 \\
155.2 & 155.2 & 155.2 & 155.2 \\
155.2 & 155.2 & 155.2 & 155.2 \\
155.0 & 155.0 & 155.1 & 155.1 \\
155.2 & 155.2 & 155.2 & 155.2 \\
155.1 & 155.2 & 155.2 & 155.2 \\
155.2 & 155.2 & 155.2 & 155.2 \\
155.2 & 155.2 & 155.2 & 155.2 \\
155.1 & 155.2 & 155.2 & 155.2 \\
155.2 & 155.2 & 155.2 & 155.2 \\
155.2 & 155.2 & 155.2 & 155.2 \\
155.2 & 155.2 & 155.2 & 155.2 \\
155.2 & 155.2 & 155.2 & 155.2 \\
155.2 & 155.2 & 155.2 & 155.2 \\
155.2 & 155.2 & 155.2 & 155.2 \\
155.2 & 155.2 & 155.2 & 155.2 \\
155.2 & 155.2 & 155.2 & 155.2 \\
155.2 & 155.2 & 155.2 & 155.2 \\
155.2 & 155.2 & 155.2 & 155.2 \\
155.2 & 155.2 & 155.2 & 155.2 \\
155.2 & 155.2 & 155.2 & 155.2 \\
155.2 & 155.2 & 155.2 & 155.2 \\
155.2 & 155.2 & 155.2 & 155.2 \\
155.2 & 155.2 & 155.2 & 155.2 \\
155.2 & 155.2 & 155.2 & 155.2 \\
155.2 & 155.2 & 155.2 & 155.2 \\
155.2 & 155.2 & 155.2 & 155.2 \\
155.2 & 155.2 & 155.2 & 155.2 \\
155.2 & 155.2 & 155.2 & 155.2 \\
155.2 & 155.2 & 155.2 & 155.2 \\
155.2 & 155.2 & 155.2 & 155.2 \\
\hline & & &
\end{tabular}

\begin{tabular}{cccc}
\hline \multicolumn{4}{c}{$\left(n, p_{1}\right)=(20,0.5)$} \\
0.1 & 0.15 & 0.2 & 0.25 \\
\hline 328.2 & 328.2 & 328.2 & 328.2 \\
328.0 & 328.0 & 328.0 & 328.0 \\
329.6 & 329.4 & 329.2 & 329.0 \\
327.9 & 327.9 & 328.0 & 328.0 \\
328.0 & 328.0 & 328.0 & 328.0 \\
328.0 & 328.0 & 328.0 & 328.0 \\
328.1 & 328.0 & 328.0 & 328.0 \\
328.0 & 328.0 & 328.0 & 328.0 \\
328.1 & 328.1 & 328.0 & 328.0 \\
328.0 & 328.0 & 328.0 & 328.0 \\
328.1 & 328.1 & 328.0 & 328.0 \\
328.0 & 328.0 & 328.0 & 328.0 \\
328.0 & 328.0 & 328.0 & 328.0 \\
328.1 & 328.1 & 328.0 & 328.0 \\
328.4 & 328.3 & 328.2 & 328.2 \\
328.0 & 328.0 & 328.0 & 328.0 \\
328.0 & 328.0 & 328.0 & 328.0 \\
328.0 & 328.0 & 328.0 & 328.0 \\
328.1 & 328.1 & 328.0 & 328.0 \\
327.3 & 327.6 & 327.8 & 328.0 \\
328.0 & 328.0 & 328.0 & 328.0 \\
328.0 & 328.0 & 328.0 & 328.0 \\
328.0 & 328.0 & 328.0 & 328.0 \\
328.1 & 328.0 & 328.0 & 328.0 \\
328.1 & 328.1 & 328.0 & 328.0 \\
328.1 & 328.1 & 328.0 & 328.0 \\
328.1 & 328.1 & 328.1 & 328.1 \\
328.0 & 328.0 & 328.0 & 328.0 \\
328.1 & 328.0 & 328.0 & 328.0 \\
328.0 & 328.0 & 328.0 & 328.0 \\
328.0 & 328.0 & 328.0 & 328.0 \\
\hline & & &
\end{tabular}


Table 4: Some standardized continuous kernels

\begin{tabular}{ccc}
\hline Kernel & Domain & $K(x)$ \\
\hline Parabolic & {$[-\sqrt{5}, \sqrt{5}]$} & $\frac{3}{4 \sqrt{5}}\left(1-\frac{1}{5} x^{2}\right)$ \\
Biweight & {$[-\sqrt{7}, \sqrt{7}]$} & $\frac{15}{16 \sqrt{7}}\left(1-\frac{x^{2}}{7}\right)^{2}$ \\
Triweight & {$[-3,3]$} & $\frac{35}{96}\left(1-\frac{x^{6}}{3^{6}}-\frac{3 x^{2}}{9}+\frac{3 x^{4}}{3^{4}}\right)$ \\
Cosine & {$\left[-\frac{1}{\sqrt{1-\frac{8}{\pi^{2}}}}, \frac{1}{\sqrt{1-\frac{8}{\pi^{2}}}}\right]$} & $\sqrt{\frac{\pi^{2}}{16}-\frac{1}{2}} \cos \left(\sqrt{\frac{\pi^{2} x^{2}}{4}-2 x^{2}}\right)$ \\
Normal & $(-\infty, \infty)$ & $\frac{e^{-x^{2} / 2}}{\sqrt{2 \pi}}$ \\
\hline
\end{tabular}


Table 5: ARL values of the C-WSR EWMA chart for parameters $\lambda=0.2$, $K=2.7$, for $\sigma=0.2, n \in\{7,13,15,18,20,25\}$ and for the kernels listed in Table 4
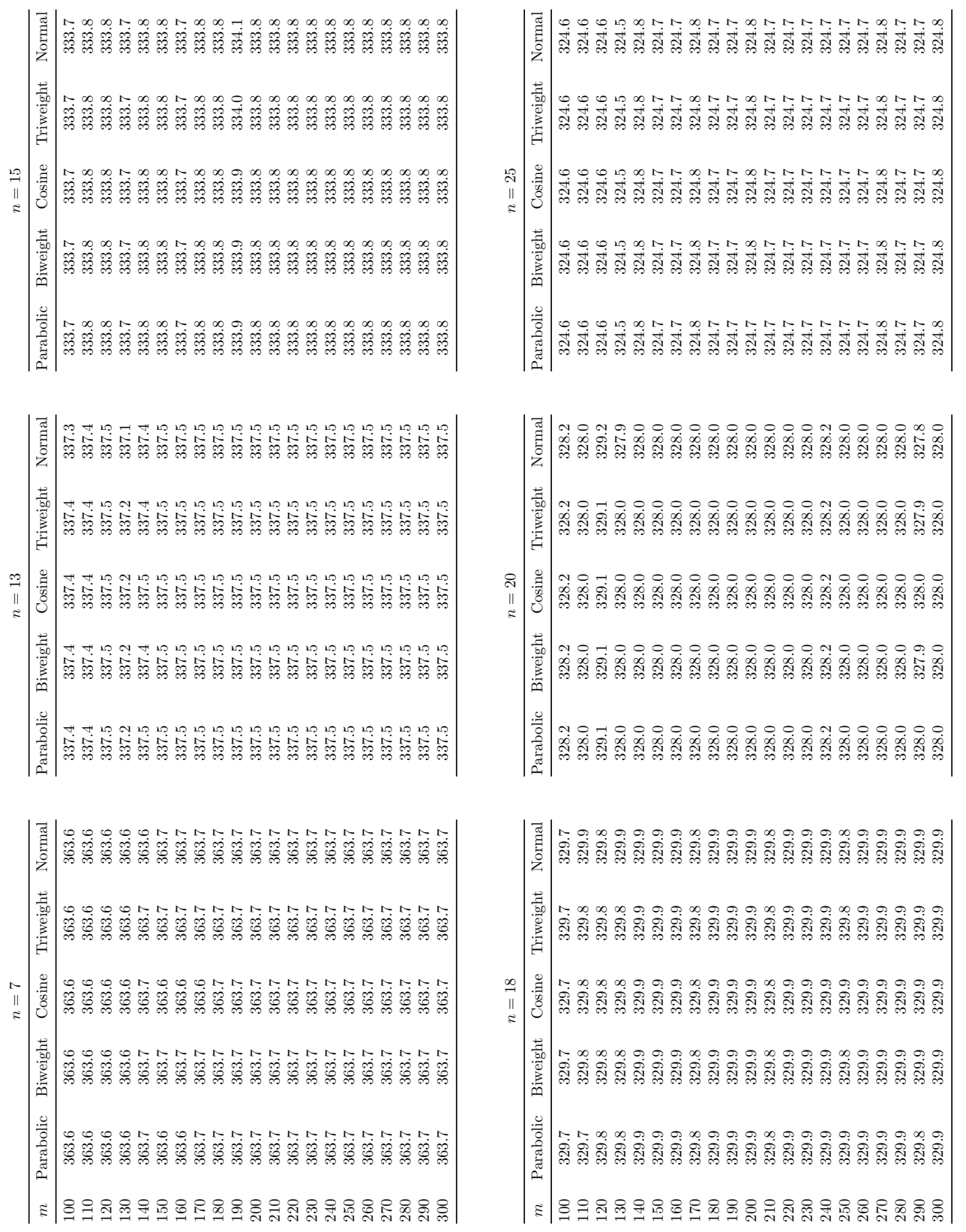
Table 6: Optimal combinations of $\left(\lambda^{*}, K^{*}\right)$ for the C-WSR EWMA chart along with the corresponding $\mathrm{ARL}_{1}$ values

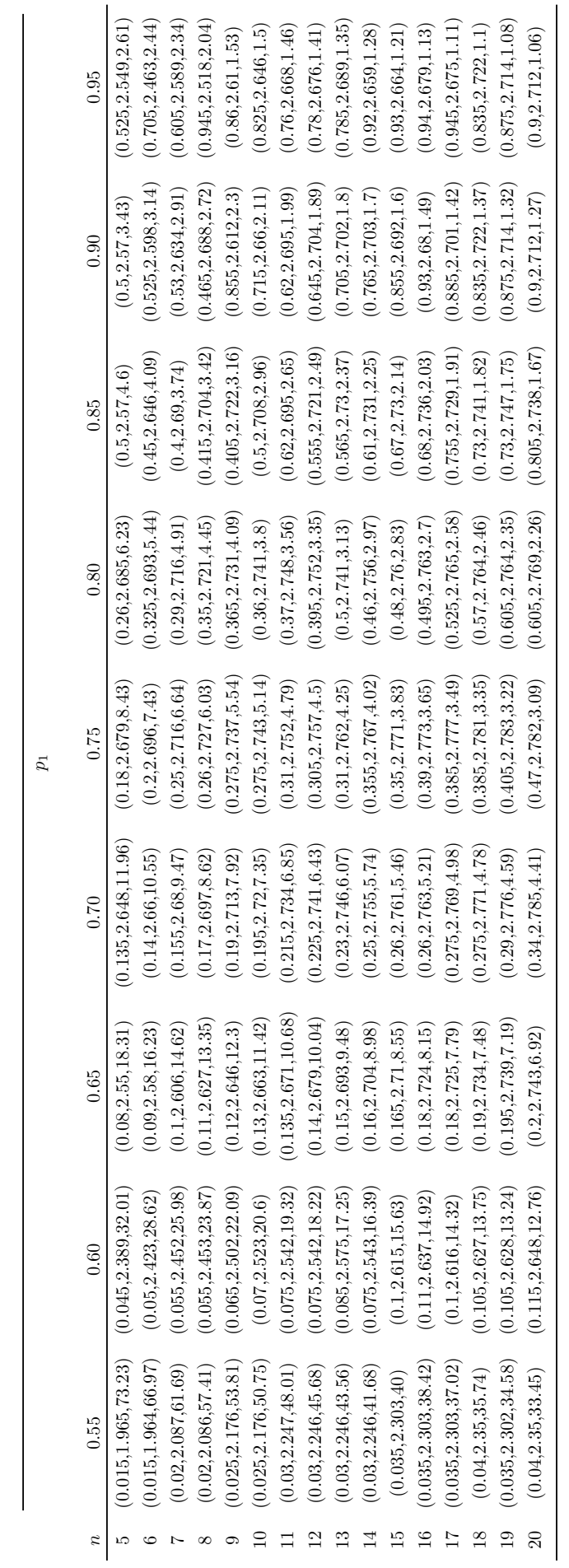


Table 7: Radial error example: Phase II sample of $t=1, \ldots 10$ subgroups of size $n=20$ and the corresponding values for $\mathrm{SR}_{t}, \mathrm{SR}_{t}^{*}$ and $Z_{t}^{*}$

\begin{tabular}{ccccccccccc}
\cline { 2 - 10 }$t$ & 1 & 2 & 3 & 4 & 5 & 6 & 7 & 8 & 9 & 10 \\
\hline 1 & 0.289 & 0.380 & 0.483 & 0.288 & 0.544 & 0.390 & 0.567 & 0.512 & 0.433 & 0.168 \\
2 & 0.447 & 0.599 & 0.207 & 0.317 & 0.256 & 0.433 & 0.218 & 0.329 & 0.432 & 0.674 \\
3 & 0.081 & 0.368 & 0.435 & 0.216 & 0.246 & 0.229 & 0.623 & 0.455 & 0.394 & 0.616 \\
4 & 0.954 & 0.537 & 0.621 & 0.513 & 1.540 & 0.609 & 0.801 & 1.080 & 1.069 & 0.954 \\
5 & 0.316 & 0.237 & 0.286 & 0.879 & 0.190 & 0.104 & 0.570 & 0.448 & 0.269 & 0.746 \\
6 & 0.342 & 0.378 & 0.287 & 0.328 & 0.589 & 0.233 & 0.255 & 0.119 & 0.284 & 0.499 \\
7 & 0.370 & 0.391 & 0.525 & 0.459 & 1.280 & 0.470 & 0.482 & 0.032 & 0.525 & 0.628 \\
8 & 0.352 & 0.264 & 0.759 & 0.154 & 0.256 & 0.426 & 0.363 & 0.310 & 0.303 & 0.316 \\
9 & 0.305 & 0.352 & 0.468 & 0.224 & 0.739 & 0.234 & 0.171 & 0.250 & 0.308 & 0.431 \\
10 & 0.603 & 0.363 & 0.628 & 0.314 & 0.029 & 0.436 & 0.207 & 0.553 & 0.645 & 0.122 \\
\hline
\end{tabular}

\begin{tabular}{|c|c|c|c|c|c|c|c|c|c|c|c|c|c|}
\hline \multirow[b]{2}{*}{$t$} & \multicolumn{10}{|c|}{$X_{t, j}$} & \multirow[b]{2}{*}{$\mathrm{SR}_{t}$} & \multirow[b]{2}{*}{$\mathrm{SR}_{t}^{*}$} & \multirow[b]{2}{*}{$Z_{t}^{*}$} \\
\hline & 11 & 12 & 13 & 14 & 15 & 16 & 17 & 18 & 19 & 20 & & & \\
\hline 1 & 0.128 & 0.428 & 0.081 & 0.575 & 0.396 & 0.574 & 0.730 & 0.407 & 0.367 & 0.452 & 45 & 45.0453 & 15.3154 \\
\hline 2 & 0.233 & 0.570 & 0.748 & 0.364 & 0.372 & 0.798 & 0.218 & 0.405 & 0.060 & 0.632 & 27 & 26.9181 & 19.2603 \\
\hline 3 & 0.116 & 0.611 & 0.666 & 0.262 & 0.410 & 0.234 & 0.692 & 0.719 & 1.033 & 0.376 & 44 & 43.8633 & 27.6253 \\
\hline 4 & 0.852 & 0.425 & 1.389 & 0.794 & 1.081 & 0.900 & 0.521 & 0.576 & 0.761 & 0.535 & 210 & 210.1701 & 89.6906 \\
\hline 5 & 0.344 & 0.191 & 0.366 & 0.315 & 0.408 & 0.522 & 0.598 & 0.232 & 0.671 & 0.448 & 0 & -0.0792 & 59.1688 \\
\hline 6 & 0.410 & 0.668 & 0.385 & 0.594 & 0.390 & 0.265 & 0.409 & 0.434 & 0.628 & 0.316 & -11 & -10.8336 & 35.3680 \\
\hline 7 & 0.686 & 0.584 & 0.300 & 0.245 & 0.555 & 0.113 & 0.194 & 0.932 & 0.597 & 0.523 & 84 & 84.0995 & 51.9367 \\
\hline 8 & 0.807 & 0.235 & 0.173 & 0.183 & 1.105 & 0.068 & 0.368 & 0.736 & 0.097 & 0.060 & -54 & -53.9357 & 15.9401 \\
\hline 9 & 0.092 & 0.326 & 0.455 & 0.569 & 0.354 & 0.475 & 0.530 & 0.312 & 0.102 & 0.651 & -31 & -31.0695 & 0 \\
\hline 10 & 0.759 & 0.296 & 0.691 & 0.425 & 0.441 & 0.323 & 0.287 & 0.310 & 0.194 & 0.582 & 18 & 17.9971 & 6.1190 \\
\hline
\end{tabular}



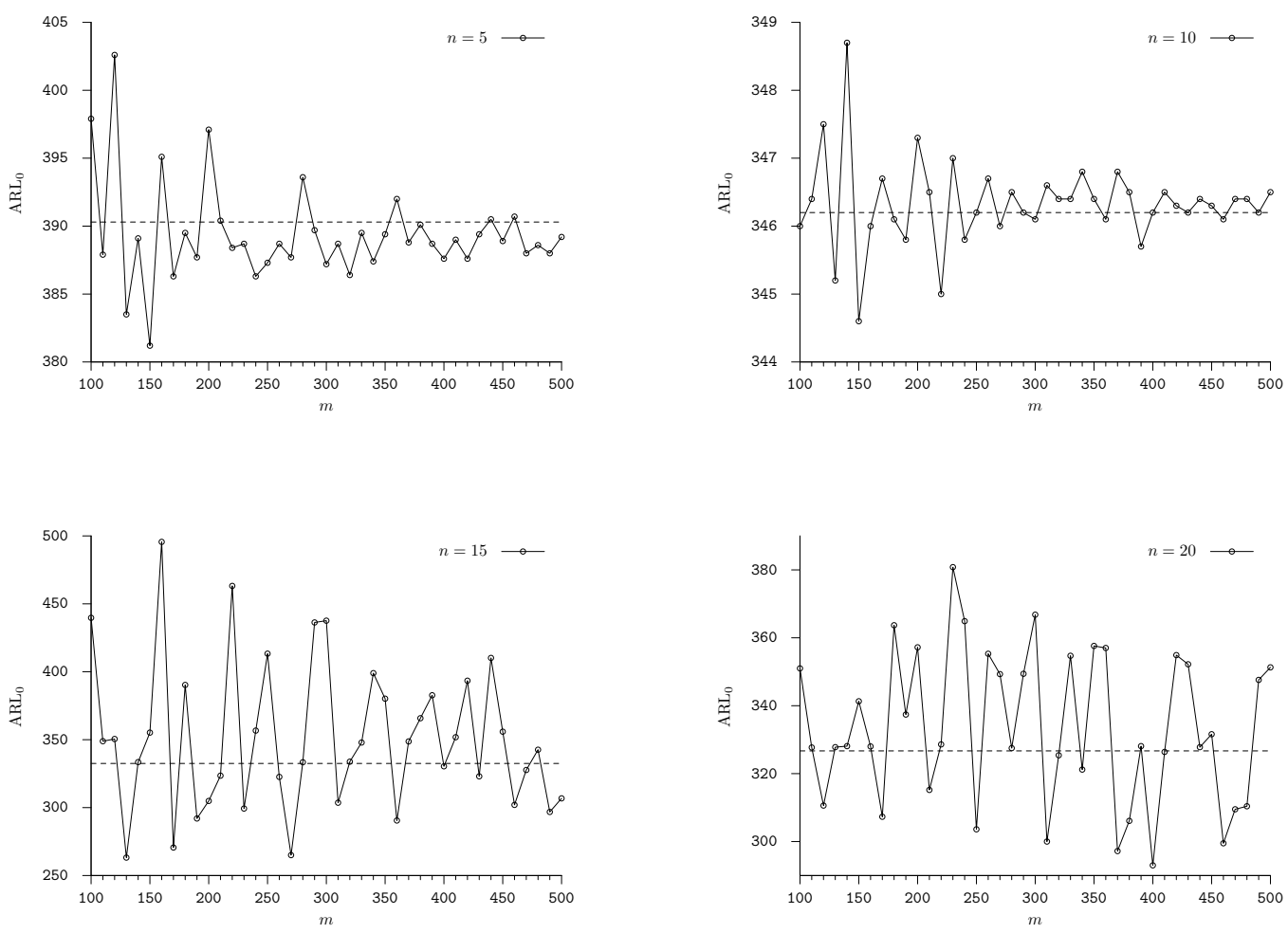

Figure 1: $\mathrm{ARL}_{0}$ (plain lines) in function of the number of sub-intervals $m \in$ $\{100,110, \ldots, 500\}$ for the upper-sided WSR EWMA chart with parameters $(\lambda=0.2, K=2.7)$ and $n \in\{5,10,15,20\}$ using the standard Markov Chain method 


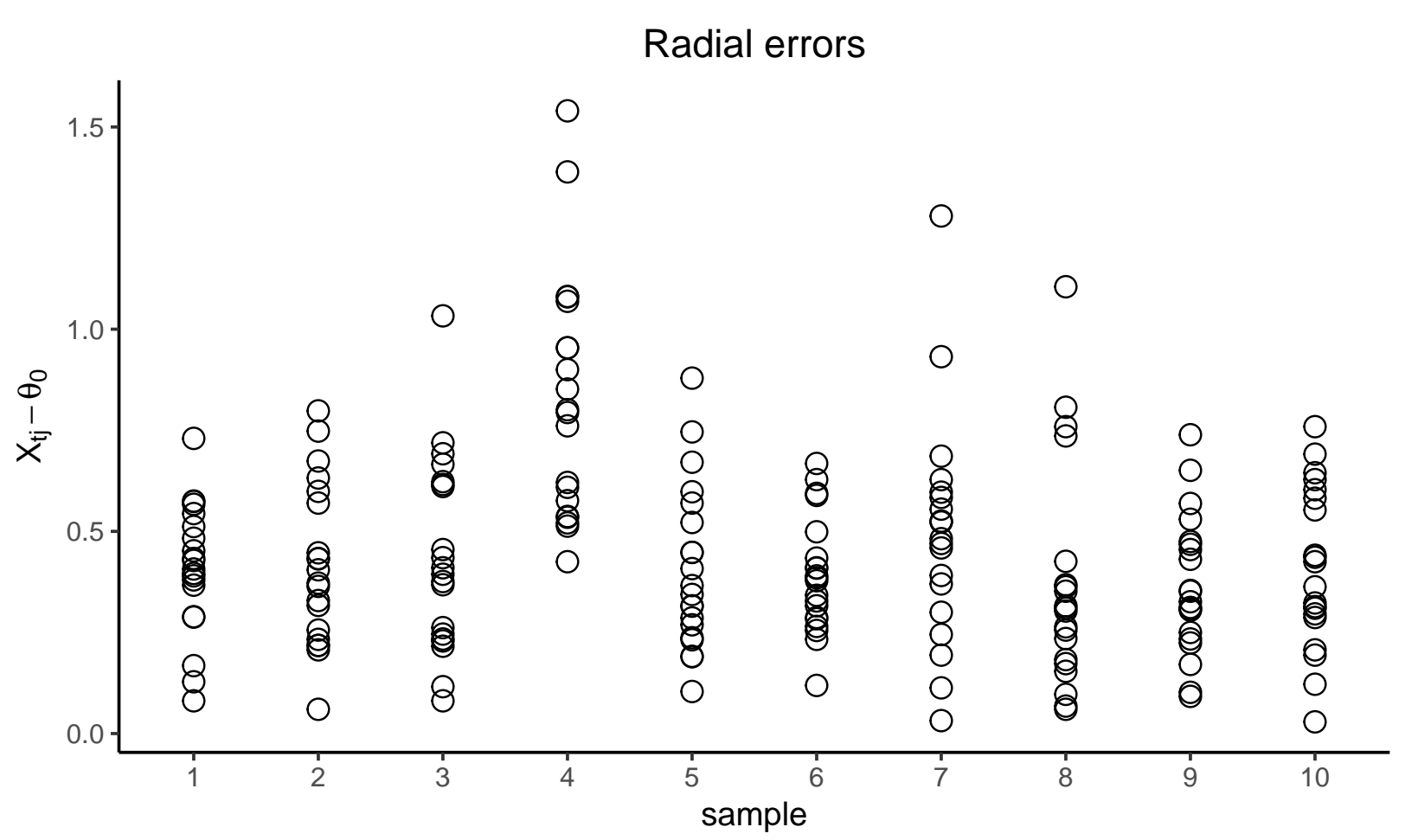

Figure 2: Radial error example: individual value plot of the observations 


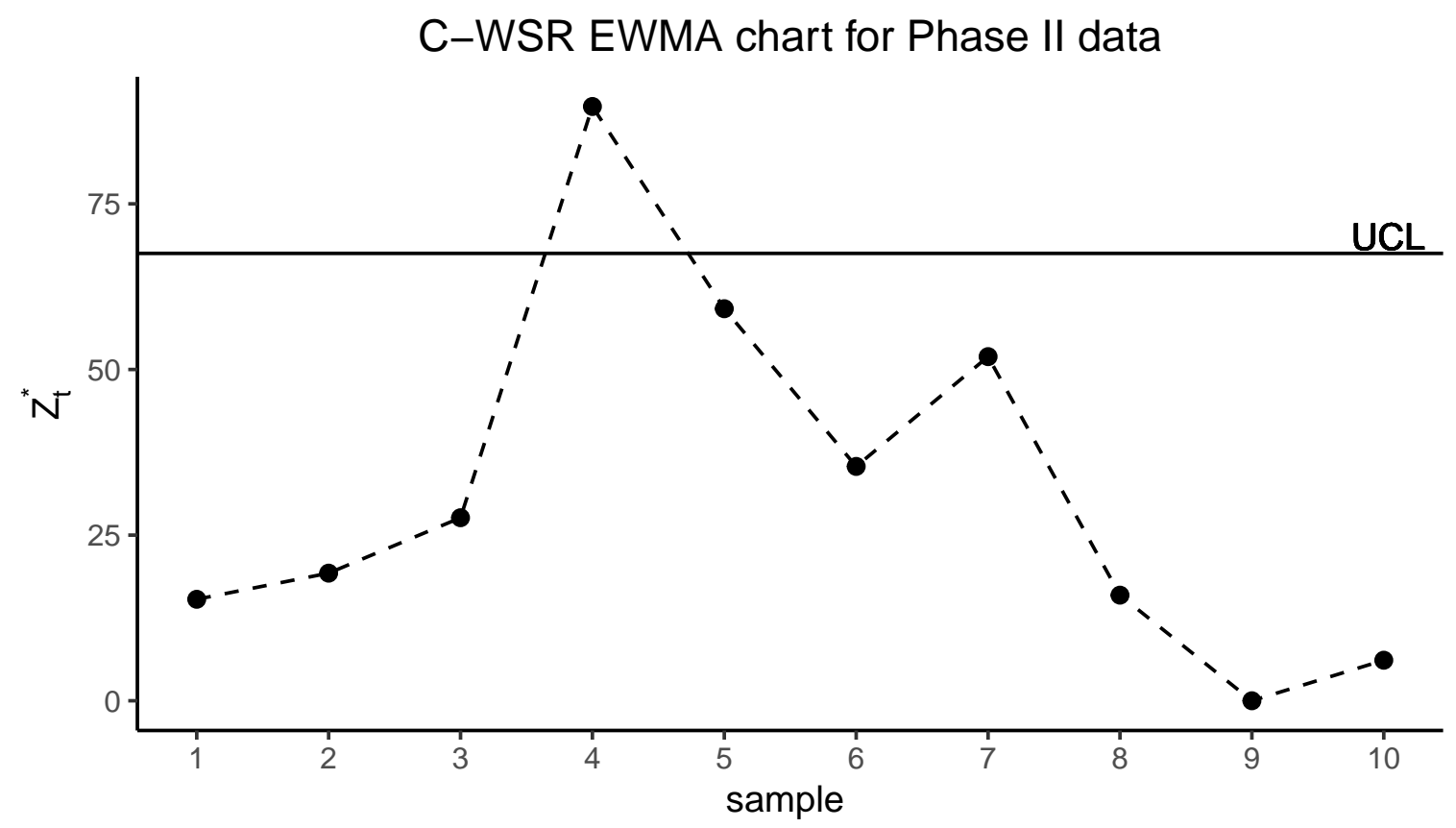

Figure 3: Radial error example: the C-WSR EWMA chart for the Phase II data presented in Table 7 